\title{
Análisis de reducción de ruido eléctrico en MOSFETS tipo SI y SiC mediante disparadores ópticos
}

\section{Electric noise reduction analysis in MOSFET Si and SiC by optic drivers}

\author{
MARTÍNEZ-HERNÁNDEZ, Moisés Agustín†*, CAMPOS-CRUZ, Luis David, SALINAS-RICO, \\ Andrés y CARDENAS-PEREZ, David
}

Universidad Autónoma de Querétaro

ID $1^{\mathrm{er}}$ Autor: Martínez-Hernández, Moisés Agustín / ORC ID: 0000-0001-5495-5185, CVU CONACYT ID: 370689

ID $1^{\text {er }}$ Coautor: Luis David, Campos-Cruz / ORC ID: 0000-0003-2419-3823, CVU CONACYT ID: 1000651

ID $2^{\text {do }}$ Coautor: Andrés, Salinas-Rico / ORC ID: 0000-0002-3816-7864, CVU CONACYT ID: 1000650

ID $3^{\text {er }}$ Coautor: David, Cardenas-Perez / ORC ID: 0000-0002-2271-5571, CVU CONACYT ID: 1000510

DOI: $10.35429 /$ JTEN.2019.12.3.26.32

Recibido 04 de Octubre, 2019, Aceptado, 03 de Diciembre, 2019

\begin{abstract}
Resumen
Las actuales demandas en el desarrollo de electrónica de potencia, enfocadas a redes eléctricas inteligentes y sistemas de cargas para autos eléctricos, genera demanda y requisitos para la fabricación de dispositivos y para el material semiconductor utilizado. La ya conocida tecnología de silicio no es capaz de soportar este tipo de condiciones, debido a que estos no son capaces de funcionar a altas temperaturas. Es por eso que los materiales similares, como el carburo de silicio (SiC), generan un gran interés por las propiedades eléctricas que presenta en relación con otros semiconductores. Sin embargo, las pérdidas por su rápida conmutación hacen que los dispositivos $\mathrm{SiC}$ no puedan llevarse a conmutaciones de altas frecuencias, que son requisito para implementaciones en convertidores de alta potencia. En el presente trabajo se analizan las diferencias en el funcionamiento de los MOSFET de $\mathrm{SiC}$ y $\mathrm{Si}$, así como ver la eficiencia que se obtiene al proponer una conmutación por drivers ópticos.
\end{abstract}

MOSFET, Análisis, Opto-drivers

\begin{abstract}
The current demands in the development of applications in power electronics, centered on intelligent electrical networks, power converters and charging systems for electric cars, to mention some of them, generate demands and requirements for the manufacture of devices and for the semiconductor material used. The already known silicon technology is not able to withstand this type of conditions, since they can not operate at high temperatures. That is why similar materials, such as silicon carbide $(\mathrm{SiC})$, generate a great interest for the electrical properties that it presents in relation to other semiconductors. However, the losses due to their rapid switching mean that $\mathrm{SiC}$ devices can not operate at high frequency, which is a requirement for implementations in high power converters. In the present work, the differences in the operation of the $\mathrm{SiC}$ and $\mathrm{Si}$ MOSFETs are analyzed, as well as the efficiency obtained when proposing a switching through optical controllers.
\end{abstract}

MOSFET, Analysis, Opto-drivers

Citación: MARTÍNEZ-HERNÁNDEZ, Moisés Agustín, CAMPOS-CRUZ, Luis David, SALINAS-RICO, Andrés y CARDENAS-PEREZ, David. Análisis de reducción de ruido eléctrico en MOSFETS tipo SI y SiC mediante disparadores ópticos. Revista de Ingeniería Tecnológica. 2019 3-12: 26-32

\footnotetext{
* Correspondencia del Autor (Correo electrónico: moisesmartinez050288@ gmail.com)

$\dagger$ Investigador contribuyendo como primer autor.
} 


\section{Introducción}

Desde su introducción a mediados del siglo XX, el silicio se convirtió en el material semiconductor con mayor presencia en la fabricación de dispositivos electrónicos. Su abundancia en el planeta, la disponibilidad de métodos efectivos para su extracción, purificación y cristalización, así como su característica de alta resistencia dieléctrica para dispositivos de potencia, son algunas de las propiedades que hacen que el silicio prevalezca como semiconductor sobre otros materiales (Casady \& Johnson. 1996).

Durante los últimos años, la investigación en el campo de los semiconductores ha permitido la implementación de soluciones basadas en Carburo de Silicio (SiC), capaces de proporcionar una mayor eficiencia en el manejo de altas frecuencias de conmutación, así como la capacidad de trabajar con una densidad de potencia mucho mayor al que operan los dispositivos de silicio tradicionales (Vacca, 2017). La figura 1 describe la eficiencia en la administración de energía del $\mathrm{SiC}$ antes del Si. Gracias a su capacidad para minimizar las pérdidas de energía, mejorar la eficiencia hasta un $10 \%$ y disminuir hasta 5 veces el espacio muerto en los dispositivos, el $\mathrm{SiC}$ representa un paso evolutivo considerable en el campo de los semiconductores (Friedrichs \& Buschkühle. 2016).

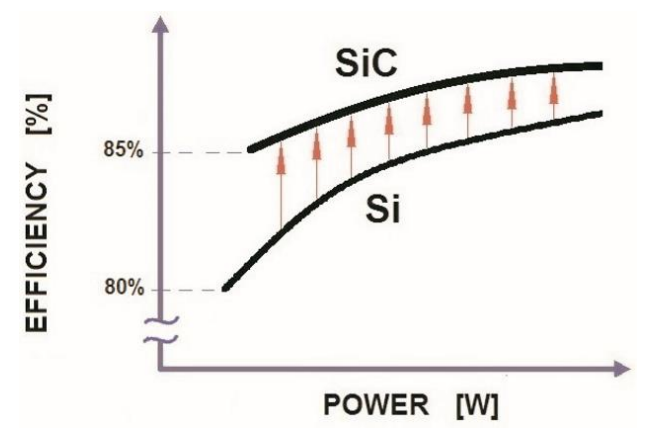

Figura 1 Eneficienia energética de semiconductores SiC y Si (Vacca, 2017)

Asimismo, la superioridad de la tecnología $\mathrm{SiC}$ como semiconductor se debe a propiedades tales como el nivel de conductividad térmica que posee, superior al de Si. Asi mismo, la corriente de fuga, un factor que determina en gran medida el ancho de banda de un semiconductor, es considerablemente más bajo que el de Si (Biela, et al. 2011).
Además, los dispositivos que implementan $\mathrm{SiC}$ superan las soluciones tradicionales de $\mathrm{Si}$ cuando funcionan a temperaturas superiores a $250^{\circ} \mathrm{C}$, lo que le permite operar en condiciones con altas temperaturas de funcionamiento, alta potencia, alta frecuencia y alta radiación (Vaculik, 2013). La Tabla 1 muestra una comparación de las propiedades más importantes de la tecnología $\mathrm{SiC} 4 \mathrm{H}$ junto con $\mathrm{Si}$.

\begin{tabular}{|l|r|r|}
\hline Propiedad & \multicolumn{2}{c|}{ Semiconductor } \\
\hline Ancho de banda energetico $[\mathrm{eV}]$ & 1.1 & SiC (4H) \\
\hline Mobilidad electronica $\left[\mathrm{cm}^{2} / \mathrm{V} \mathrm{s}\right]$ & 1400 & 900 \\
\hline $\begin{array}{l}\text { Campo de ruptura electrico } \\
{[\mathrm{kV} / \mathrm{cm}]}\end{array}$ & 300 & 2400 \\
\hline Conductividad termica $[\mathrm{W} / \mathrm{cmK}]$ & 1.5 & 4 \\
\hline
\end{tabular}

Tabla 1 Propiedades de semiconductores SiC y Si

$\mathrm{Si}$ bien la implementación de dispositivos con tecnología $\mathrm{SiC}$ garantiza los avances mencionados, su evolución está en constante desarrollo debido a varios factores. El más importante de ellos es el empaquetado tradicional de los dispositivos, que limita el uso de los beneficios ofrecidos por el SiC. En este sentido, es interesante desarrollar estructuras de empaque mejoradas que permitan el uso de esta tecnología en cada una de sus aplicaciones.

Por otro lado, las temperaturas de operación son considerablemente más altas en comparación con los dispositivos tradicionales. Estos están en un rango de $350^{\circ} \mathrm{C}$ a $500^{\circ} \mathrm{C}$, y es un factor deseado para aplicaciones tales como instrumentación de energía nuclear, satélites, exploración espacial o motores de turbina (Chen, et al, 2017).

Actualmente, los dispositivos que operan con $\mathrm{Si}$, continúan liderando la fabricación de sistemas de energía, esto debido al nivel de desarrollo de esta tecnología, así como a su costo de implementación. Además de esto, el costo de la aplicación de dispositivos con tecnología $\mathrm{SiC}$ es elevado en comparación con los primeros, lo que reduce su disponibilidad y uso. Comúnmente, las aplicaciones que funcionan por debajo de $30 \mathrm{KHz}$ y las tensiones de bloqueo superiores a $650 \mathrm{~V}$ son implementadas con IGBT's, mientras que las que superan los 20 $\mathrm{KHz}$ y tienen una tensión de bloqueo por debajo de $650 \mathrm{~V}$ implementan los MOSFET para su funcionamiento (Alcalá, et al, 2015). 
Este artículo tiene como objetivo realizar una comparación de las tecnologías de $\mathrm{Si}$ y $\mathrm{SiC}$, con el propósito de analizar los niveles de eficiencia y las pérdidas de energía presentes en cada uno de los dispositivos, así como analizar las diferencias al implementar los disparos por dispositivos ópticos para reducir el ruido eléctrico por conmutación.

\section{Pruebas de disparo entre semiconductores $\mathrm{SiC}$ y Si}

Para el desarrollo del presente trabajo se realizaron pruebas simples para la comprobación de las teorías y poder conocer las respuestas de cada semiconductor, aunque en las hojas de datos especifican los comportamientos de cada dispositivo, es necesario caracterizar $y$ comprender el comportamiento de estos en un ambiente de pruebas diferente y con cargas. Para las pruebas se empleo us sistema de disparo controlado por un un microcontrolador (PIC18f4550) con una señal PWM de salida con una frecuencia de $8 \mathrm{KHz}$, esto para implementar el disparo semiconductor de potencia (MOSFET y SIC-MOSFET), el circuito de disparo se desarrollo con un semiconductor BC548 en la configuración del interruptor lógico, las mediciones se capturaron con puntas flotadas (Tektronix p5200A) y una punta de corriente CA / CC (Tektronix A622). La figura 2 muestra el circuito utilizado para la activación de los dispositivos de potencia.

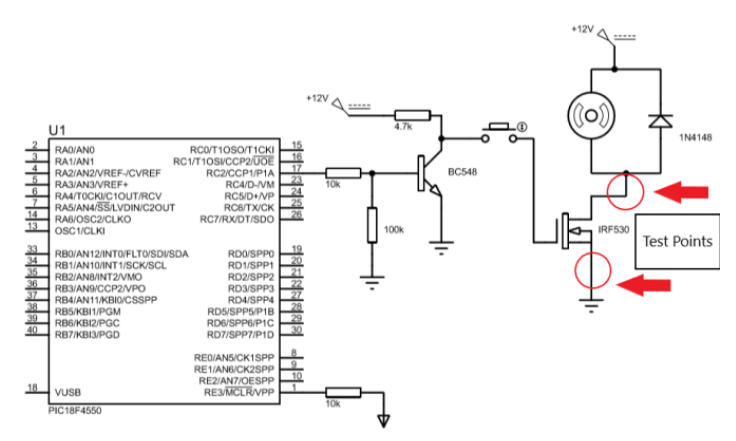

Figura 2 Circuito de prueba para captura de flancos

En las tablas 2 y 3 se muestran las principales características de los semiconductores empleados.

El circuito de activación que se utilizó para el SI-MOSFET (IRF530) es el mismo que el utilizado para el SIC-MOSFET (C2M0080120D), esto se debe a que ambos semiconductores de potencia son de tipo $\mathrm{N}$ y su configuración es similar.

\begin{tabular}{|l|c|}
\hline Drain-Source voltage & $100 \mathrm{~V}$ \\
\hline Threshold voltage in gate & $2 \mathrm{~V}(\max )$ \\
& $4 \mathrm{~V}(\min )$ \\
\hline Continuous current in drain & $14 \mathrm{~A}$ \\
\hline
\end{tabular}

Tabla 2 Carcateristicas de IRF530 (si-mosfet) (Fairchild Semiconductor, 2001)

\begin{tabular}{|l|c|}
\hline Drain-Source voltage & $1200 \mathrm{~V}$ \\
\hline Threshold voltage in gate & $\begin{array}{c}2 \mathrm{~V}(\max ) \\
4 \mathrm{~V}(\min )\end{array}$ \\
\hline Continuous current in drain & $36-24 \mathrm{~A}$ \\
\hline
\end{tabular}

Tabla 3 Caracteristicas de C2M0080120D (SiC-mosfet) (CREE, 2015)

Para observar las conmutaciones en voltaje y corriente, asi como los sobrepasos que se formaron en respuesta a la conmutación, se emplearon puntas flotadas de voltaje, esto para no dañar el equipo de medición, para observar la corriente consumida se colocó la punta de corriente CA / CD (Tektronix A622).

\section{Comparación de eficiencia}

Para verificar la funcionalidad de los MOSFETS de $\mathrm{Si}$ y $\mathrm{SiC}$, se llevó a cabo un análisis descriptivo del proceso de conmutación de ambas configuraciones, con el fin de validar conclusiones a través de la experimentación, para lo cual se utilizó un circuito digital que se activó con $5 \mathrm{~V}$.

En el caso del Si MOSFET, esto permite activar un circuito de hasta $120 \mathrm{~V}$; se colocó una carga de $12 \mathrm{~V}$, con lo cual se obtuvo un excedente de $51.2 \mathrm{~V}$, lo que genera un error de $326.6 \%$. La figura $3 \mathrm{a}$ y $3 \mathrm{~b}$ muestra el encendido de SiMOSFET (IRF530). En comparación con el MOSFET de SiC (figura 3c y 3d), alcanza un voltaje máximo de $46.4 \mathrm{~V}$, al igual que el MOSFET de Si hay un error significativo, sin embargo, esto es menor.

En términos de consumo de corriente, ambos tienen un comportamiento similar, se observa que el MOSFET de Si consume una corriente de $568 \mathrm{~mA}$, en comparación con el MOSFET de SiC, consume una corriente de 512mA. El MOSFET de SiC tiene un comportamiento similar al MOSFET de $\mathrm{Si}$, aunque la demanda de corriente es similar, el voltaje máximo obtenido es mayor con el MOSFET de Si 


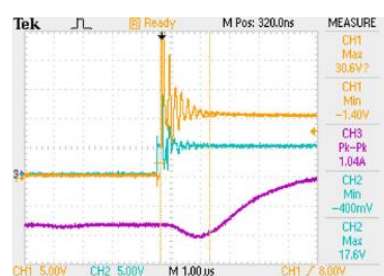

(a)

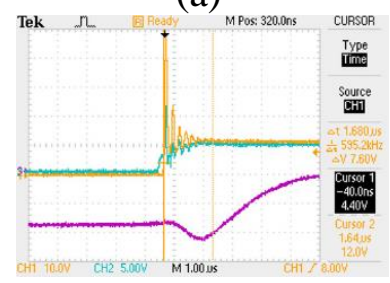

(c)

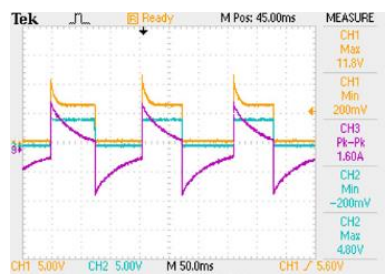

(b)

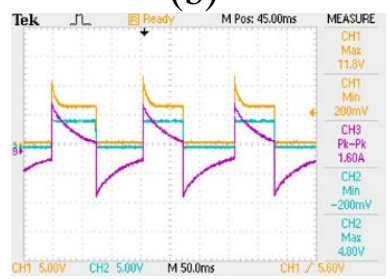

(d)
Figura 3 Pruebas de activación y desactivación

\section{a), b) Si-MOSFET \\ c), d) SiC-MOSFET}

\section{Pruebas de $\mathrm{SiC}$ con carga inductiva en aplicación de potencia}

Para obtener una mejor comprobación en los tiempos de respuesta, se procedio a realizar pruebas en los dispositivos $\mathrm{SiC}$, ante la programacion de un pulso y su complementario a $50 \mathrm{Khz}$ (generadas con un DSP TMS320F377D), para la activación de un puente $\mathrm{H}$ con un inductor y un trasformador elevador (1:8.1). Esto con el fin de observar el rendimiento de los $\mathrm{SiC}$ ante cargas inductivas y aplicaciones de potencia.

En la figura 4 se observa el diagrama del sistema de prueba, la tensión del bus de CD fue de $12 \mathrm{~V}$. La carga que se aplico fue la que se calculó para obtener la potencia máxima de $3 \mathrm{KW}$.

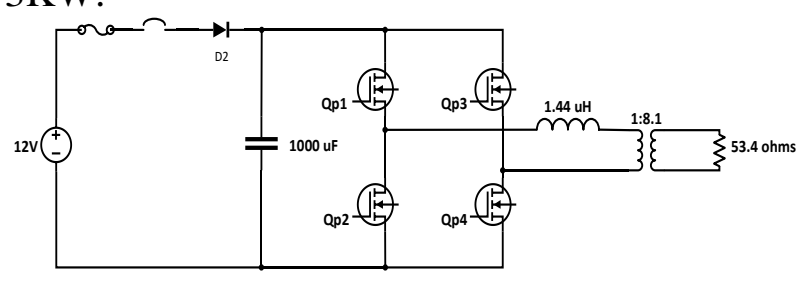

(a)

Figura 4 Diagrama de conexiones de prueba

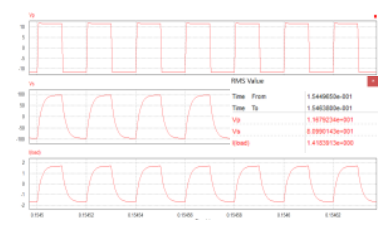

(a)

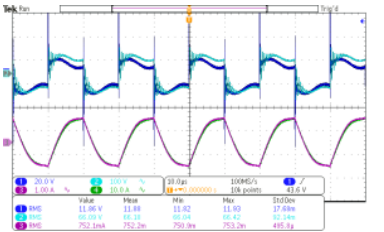

(b)
Figura 5 Señales de respuesta en pruebas del transformador e inductor (a) Simulación con parámetros implementados

(b) Señales de Osciloscopio

La figura 5a muestra las señales obtenidas al simular el sistema, donde entrega los parámetros de que se muestran en la tabla 4, se observan 3 señales que representan la tensión en el primario del transformador (Vp), la tensión en el secundario (Vs) y la corriente en la carga (iload). La figura $5 \mathrm{~b}$ muestran las mismas señales de la simulación, donde la tensión del primario se muestra con la señal azul, la tensión del secundario es la señal cian y la corriente en la carga es la señal morada.

\begin{tabular}{|l|l|}
\hline \multicolumn{2}{|c|}{ Simulación } \\
\hline Tensión en primario (Vrms) & $11.67 \mathrm{~V}$ \\
\hline Tensión en secundario (Vrms) & $80.99 \mathrm{~V}$ \\
\hline Corriente en carga (Irms) & $1.41 \mathrm{~A}$ \\
\hline \multicolumn{2}{|c|}{ Implementación } \\
\hline Tensión en primario (Vrms) & $11.86 \mathrm{~V}$ \\
\hline Tensión en secundario (Vrms) & $66.09 \mathrm{~V}$ \\
\hline Corriente en carga (Irms) & $0.752 \mathrm{~A}$ \\
\hline
\end{tabular}

Tabla 4 Parámetros de simulación e implementación

Se puede ver en la tabla una variación entre las corrientes en la carga y las tensiones del secundario, de igual manera en la figura $5 \mathrm{~b}$ se observa que la señal de tensión en el secundario presenta oscilaciones drásticas en los transitorios, esta oscilación se puede traducir como perdidas en el transformador.

Para realizar un análisis de la fuente del ruido fue necesario poner el sistema en marcha para monitorear las señales en diferentes puntos.

Como se muestra en la figura 6a, la señal del primario presenta una oscilación minima, esta oscilación se incrementa en el secundario (figura 6b) por la relación de transformación.

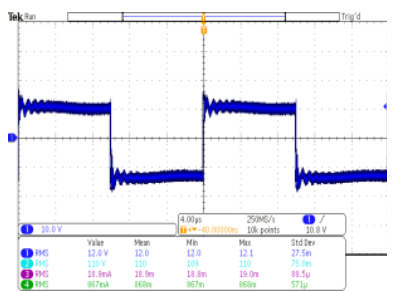

(a)

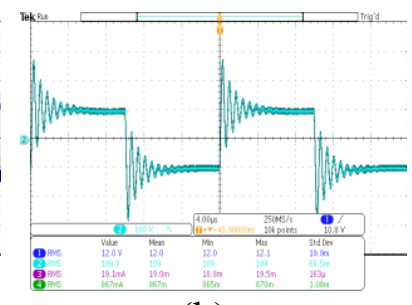

(b)
Figura 6 Señales del transformador sin carga con $12 \mathrm{~V}$ de $\mathrm{CD}$

(a) Tensión del primario. (b) Tensión del secundario 
Al observar el comportamiento de las señales del bus de $\mathrm{CD}$, se observa que la tensión (figura 6a, señal azul) presenta ligeras oscilaciones que al medirlas se presentan cada $10 \mathrm{us,}$ es decir, una frecuencia de $100 \mathrm{KHz}$.

Empleando una sonda especializada para medición de ruido, se obtiene la imagen que se muestra en la figura $7 b$, la cual fue analizada en un MOSFET del puente, donde se observan la frecuencia de conmutación de $50 \mathrm{KHz}$, además de ruido por frecuencias de $100 \mathrm{KHz}$, en la figura se observan 2 cursores posicionados, donde la diferencia entre ellos es de $0.1 \mathrm{MHz}$.

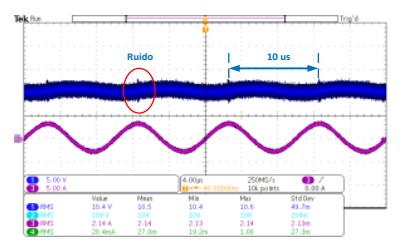

(a)

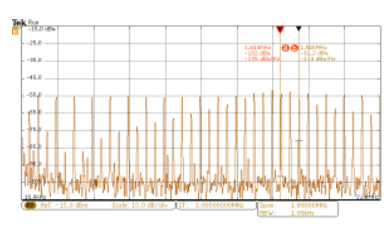

(b)
Figura 7 Señales del sistema sin carga con $12 \mathrm{~V}$ de CD

(a) Tensión del primario. (b) Tensión del secundario

Con esas señales se puede entender que el problema del ruido presente en el sistema es debido a la conmutación de los semiconductores. A pesar de las ventajas del $\mathrm{SiC}$, esta característica afectaba al conmutar a un tiempo reducido y pasar niveles de tensión altos.

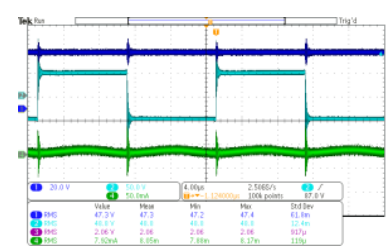

(a)

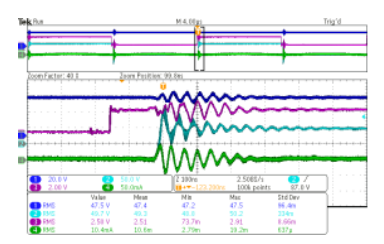

(b)
Figura 8 Oscilaciones en señales del sistema sin carga

(a) Conmutación de los MOSFET. (b) Aumento de imagen en transitorios

En la figura anterior se observa que las señales aumentan en la amplitud de las oscilaciones. La figura 8a muestra las señales de conmutación de un mosfet (cian), la tensión del bus de CD (azul) y la corriente en el bus de CD (verde), al operar a $48 \mathrm{~V}$ las oscilaciones causadas por la conmutación de los semiconductores incrementa.
La forma de mejorar estas respuestas y evitar las pérdidas del sistema, se procedio a realizar el disparo del sistema con disparadores ópticos, para reducir el ruido por conmutación.

\section{Pruebas de SiC con disparadores ópticos}

Para estas pruebas se realizaron utilizando el sistema como se muestra en la figura 9.

Los resultados que se obtuvieron, permitieron la implementación de las pruebas con el transformador e inductor.

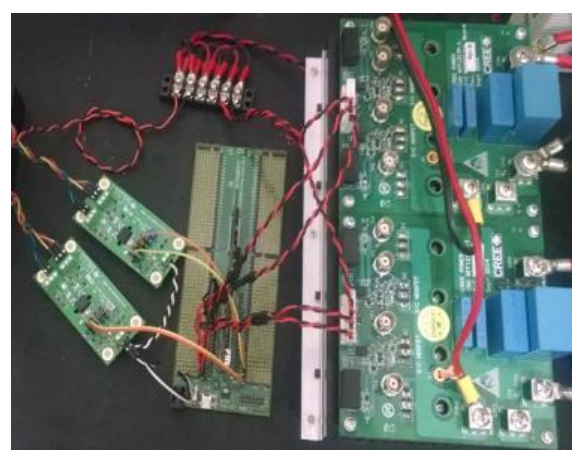

Figura 9 Pruebas en el puente SiC. Conexiones puentedrivers-DSP

En la figura 10a se observan las señales de conmutación generadas por el DSP (morada y verde), estas señales son de un PWM con su complementario. Las señales del DSP van al driver que amplifica la tensión además de ofrecer aislamiento entre la etapa de potencia y el DSP, posteriormente se miden las señales PWM en los semiconductores (azul y cian), como se muestra en las medidas del osciloscopio (figura 10a), las tensiones en los mosfets son mayores a las del DSP.

La principal ventaja de estas señales, es la ausencia de las oscilaciones que se presentaban anteriormente, esto gracias al uso de los disparadores ópticos.

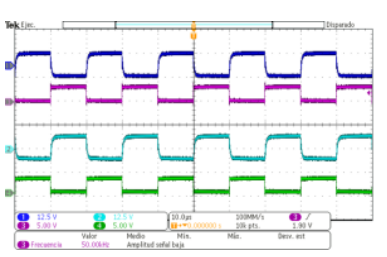

(a)

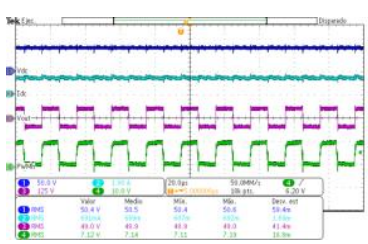

(b)
Figura 10 Señales de operación de conmutación

(a) Señales de conmutación. (b) Señales de entrada y salida 
En la figura 10b se observan la tensión en el bus de CD (azul), así como la corriente (cian), se aprecian ligeras perturbaciones, estas de igual manera, son por las conmutaciones, sin embargo, se disminuyeron considerablemente mostrando la mejora del sistema.

En la figura 11 se muestran las señales al conectar el puente $\mathrm{H}$ con el inductor $\mathrm{y}$ el transformador, para esa prueba no se puso carga en el secundario. Se puede observar en las medidas del osciloscopio que la tensión rms del primario (azul) es de $50.2 \mathrm{~V}$, mientras en el secundario (morada) es de $435 \mathrm{~V}$. La forma de onda de la corriente (cian) es similar a una triangular, donde la pendiente positiva corresponde al ciclo positivo del primario, esto representa la carga en corriente del inductor, mientras en la pendiente negativa de la señal representa la descarga, que representa la transferencia de energía hacia el secundario.

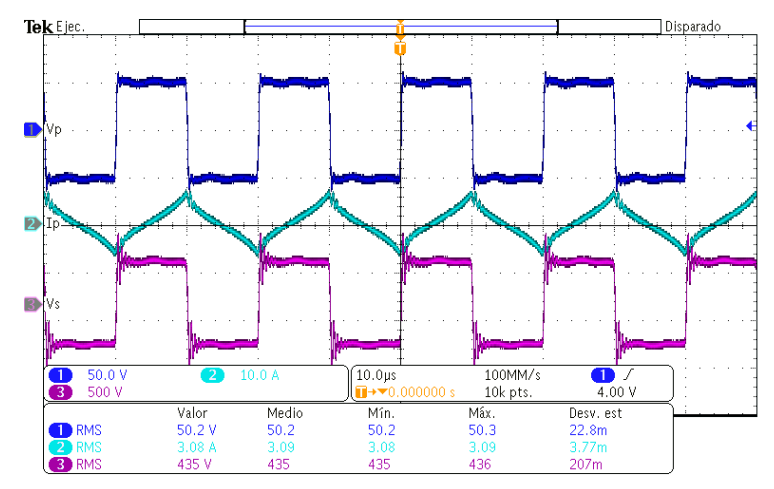

Figura 11 Señales del transformador sin carga

Las tensiones de primario y secundario, validan el funcionamiento del puente en conjunto con el inductor y el transformador construidos, asi como la mejoría en la reducción del sistema con disparadores ópticos.

\section{Conclusiones}

El análisis de eficiencia se llevó a cabo en las mismas condiciones para ambos dispositivos. El resultado de estas pruebas muestra que el tiempo de respuesta para el SiC-MOSFET es menor que el de su contraparte que opera a partir de Si. Del mismo modo, el aumento de voltaje adopta un valor pico más bajo en el proceso de conmutación. De esta manera, es posible concluir que la sustitución de dispositivos con tecnología $\mathrm{SiC}$ en aplicaciones de energía es totalmente viable, sin embargo, las diferencias con respecto a los dispositivos de $\mathrm{Si}$ son mínimas en su funcionamiento.
Al validar la eficiencia de los dispositivos $\mathrm{SiC}$ ante aplicaciones de potencia, se presentaron los problemas de oscilación en las tensiones del convertidor, lo cual fue resuelto con el uso de elementos que mejoraran su funcionalidad y así mismo dieran protección a la etapa de control, como los disparadores ópticos. Satisfactoriamente se presenta un desarrollo tecnológico que puede dar inicio a investigaciones en sistemas especializados, como un sistema de gestión distribuida con aportes novedosos que favorecezcan para la incursión de tecnologías en el sector energetico.

\section{Bibliografía}

C. Chen, F. Luo \& Y. Kang. (2017) A review of SiC power module packaging: Layout, material system and integration. CPSS Transactions on Power Electronics and Applications. vol. 2. no. 3. pp. 170-186. Sept.

CREE, C2M0080120D Silicon Carbide Power MOSFET, Rev. C, October 2015.

C. W. T. McLyman. (2004). Transformer and Inductor Design Handbook. Fourth Edition. vol. 1. CRC Press. p. 667.

D. W. Hart. (2001), Electronica de Potencia, Segunda ed., vol. 1, Pearson Educación. p. 472.

Fairchild Semiconductor. 22A, 100V, 0.064 Ohm, N-Channel Power MOSFET, 4843.1, Rev. A, February 2001.

F. Peng. (2004) A new ZVS bidirectional DCDC converter for fuel cell and battery application. IEEE Transactions on Power Electronics. vol. 19. n 1. pp. 54-65. Jun.

G. Vacca. (2017). Benefits and advantages of silicon carbide power devices over their silicon counterparts. volume 12. issue 3. April/May.

J. Alcalá. A. Aganza. D. Fraustro. V. Cárdenas M. Pacas. \& S. Charre. (2015). Characterization of the SiC MOSFET operation in hard switching compared to the trench-gate $\mathrm{Si}$ IGBT and $\mathrm{Si}$ CoolMOS TM MOSFET. in National Congress of Automatic Control, October.

J.B. Casady \& R.W. Johnson. (1996). Status of silicon carbide $(\mathrm{SiC})$ as a wide-bandgap semiconductor for high-temperature applications: a review. Solid-State Electronics. volume 39. issue 10 pages $1409-1422$. ISSN 0038-1101.

MARTÍNEZ-HERNÁNDEZ, Moisés Agustín, CAMPOS-CRUZ, Luis David, SALINAS-RICO, Andrés y CARDENAS-PEREZ, David. Análisis de reducción de ruido eléctrico en MOSFETS tipo SI y SiC mediante disparadores ópticos. Revista de Ingeniería Tecnológica. 2019 
J. Biela, M. Schweizer, S. Waffler \& J. W. Kolar. (2011). SiC versus $\mathrm{Si}$ - evaluation of potentials for performance improvement of inverter and DC-DC converter systems by $\mathrm{SiC}$ power semiconductors. IEEE Transactions on Industrial Electronics. vol. 58. no. 7. pp. 28722882. July.

P. Vaculik. (2013). The properties of $\mathrm{SiC}$ in comparison with $\mathrm{Si}$ semiconductor devices. 2013 International Conference on Applied Electronics. Pilsen. pp. 1-4.

P. Friedrichs \& M. Buschkühle. (2016). The future of power semiconductors: rugged and high performing silicon carbide transistors. Energetica India. May/June. 